\title{
Dual isotope stress Tl-201 and rest Tc-99m CZT SPECT: Are we truly leveraging CZT technology?
}

\author{
Saurabh Malhotra, MD, MPH, FASNC, a and Rami Doukky, MD, MSc, FASNC ${ }^{\mathrm{b}, \mathrm{c}}$ \\ a Division of Cardiovascular Medicine, Jacobs School of Medicine and Biomedical Sciences, \\ University at Buffalo, Buffalo, NY \\ b Division of Cardiology, Cook County Health and Hospitals Systems, Chicago, IL \\ c Division of Cardiology, Rush University Medical Center, Chicago, IL
}

Received Feb 15, 2018; accepted Feb 15, 2018

doi: $10.1007 / \mathrm{s} 12350-018-1242-7$

\section{See related article, pp. 1269-1279}

Conventional Anger cameras with NaI technology form the backbone of majority of the radionuclide imaging laboratories. The design of the conventional Anger camera suffers from major limitations, such as low photon sensitivity and low inherent spatial resolution, requiring relatively higher doses of radioisotope and longer imaging times to provide diagnostic quality images. The introduction of solid-state technology has revolutionized myocardial perfusion imaging (MPI). The majority of the solid-state cameras use CadmiumZinc-Telluride (CZT) semiconductor for photon detection. These semiconductors combine the functions of scintillation crystal and photomultiplier tubes and produce current directly in response to the photons. CZT crystals can process $>10$ million photons $/$ second $/ \mathrm{mm}^{2}$ and thus provide a greater photon sensitivity with improved energy resolution compared to conventional $\mathrm{NaI}$ crystal. This higher energy resolution conferred by CZT cameras translates into improved image resolution and for diagnostic imaging to be performed with low to very low dose of radiotracer. Results from the MILLISIEVERT study highlight the fact that "ultra-low-dose", imaging using only $3.5 \mathrm{mCi}$ of Tc-99m (1 mSv) can be performed with solid-state cameras, with a superior image quality and similar diagnostic accuracy when compared to standard anger cameras. ${ }^{1}$ The greater

Reprint requests: Rami Doukky, MD, MSc, FASNC, Division of Cardiology, Cook County Health and Hospitals Systems, 1901 W. Harrison St., Suite 3620, Chicago, IL 60612; rdoukky@ cookcountyhhs.org J Nucl Cardiol 2019;26:1280-3.

1071-3581/\$34.00

Copyright (C) 2018 American Society of Nuclear Cardiology. sensitivity of the CZT cameras allows for ultra-fast imaging (1-2 minutes) with standard doses of radioisotopes, thus providing the ability to improve laboratory throughput. Novel protocols for SPECT imaging with varying combination of isotope type, dose, and imaging times have been reported and can provide laboratories employing CZT technology significant flexibility in performing appropriate, patient-centered imaging with high diagnostic accuracy.

The higher sensitivity, improved image resolution and reduction in radiation have translated beyond Tc$99 \mathrm{~m}$ tracers, with the provision of performing 'lower' dose dual isotope protocols as well. Though not recommended, ${ }^{2}$ reports of imaging with dual isotope protocols with CZT cameras, at half the dose when compared to conventional Anger cameras, have been reported. In a study of 102 patients undergoing stress/ rest Tc-99m MPI on a CZT camera, Imbert et al. performed additional Tl-201 imaging at rest. ${ }^{3}$ The authors reported a comparable accuracy for detection of myocardial ischemia when comparing data from dual isotope stress/rest imaging ( $\mathrm{AUC}=0.83 \pm 0.03$ ) vs. single isotope stress/rest imaging ( AUC $=0.81 \pm 0.03$ ). Importantly, the authors employed an ultra-low dose Tc$99 \mathrm{~m}(1 \mathrm{mSv})$ for stress, and low-dose rest Tc-99m (3.3 $\mathrm{mSv}$ ) or a low-dose rest Tl-201 (7.3 mSv). The authors further reported that close to $40 \%$ of studies at their institution are performed as stress-only, suggesting that effective stress MPI with CZT can be performed at 1 $\mathrm{mSv}$ of radiation exposure. Other studies have also reported the ability to perform of a diagnostic SPECT MPI with low or ultra-low radioisotope dose on a CZT camera. $^{4-6}$

In this issue of the Journal, Barone-Rochette et. al, report the performance of a dual isotope stress Tl-201/ rest Tc-99m protocol employing Discovery NM 530c CZT camera (GE Healthcare) in 54 patients being 
evaluated for coronary artery disease $(\mathrm{CAD}){ }^{7} \quad$ All patients enrolled in this study were scheduled to undergo a clinically indicated invasive coronary angiogram (ICA), with fractional flow reverse (FFR) assessment for intermediate stenosis (50-90\%). Each patient underwent Tl-201 stress-first with supine (gated) and prone imaging, followed by a supine Tc-99m resting SPECT (gated). The lowest possible, weight-based, dose of the isotopes used was $2 \mathrm{mCi}$ for Tl-201 and $8 \mathrm{mCi}$ for Tc$99 \mathrm{~m}$, which approximates to $11.5 \mathrm{mSv}$ of radiation exposure $(8.8 \mathrm{mSv}$ if stress-first or stress-only imaging is performed). ${ }^{8}$ The authors report a good sensitivity of this dual isotope protocol to detect hemodynamically significant CAD, though with a suboptimal specificity. Despite similar dosimetry when compared to a single isotope Tc-99m protocol on a conventional Anger camera, dual isotope imaging confers a few advantages. The improved sensitivity and spatial resolution of the CZT cameras allowed for faster acquisition of high quality stress images with relatively lower Tl-201 dose (2 $\mathrm{mCi}$ ) compared to $2.5-3.5 \mathrm{mCi}$ administered with conventional Anger camera, yielding relatively lower effective dose of radiation. ${ }^{8}$ Since Tl-201 stress imaging is performed first, Tc-99m down-scatter in the Tl-201 energy window is avoided. As reported in the current study, the imaging times were approximately 20 minutes for the entire study. This higher throughput is a definitive advantage over conventional rest/stress single isotope protocols. The second, theoretical, advantage pertains to the use of Tl-201 itself, which has a superior extraction fraction, thus potentially allowing for detection of perfusion defects with a greater sensitivity. Per Barone-Rochette et al., this forms the main premise of performing dual isotope CZT SPECT.

In contemporary practice of nuclear cardiology, we are continuously striving to provide excellent diagnostic capabilities, and application CZT technology is a big step in this direction. However, the use of dual isotope protocols seems retrogressive in this regard and does not fully mine the flexibility conferred by CZT cameras. As nuclear cardiologists, our goal is to provide accurate diagnostic, prognostic, and therapeutic guidance to our referral base, while maintaining laboratory efficiency, improving image quality, and reducing radiation exposure. Imaging with Tl-201 does offer the theoretical advantage of better sensitivity due to greater extraction fraction when compared to Tc-99m, though this presumed diagnostic superiority, reported in animal studies, ${ }^{9,10}$ has not been consistently documented in clinical scenarios. ${ }^{11-14}$ In a small study $(n=38)$ of

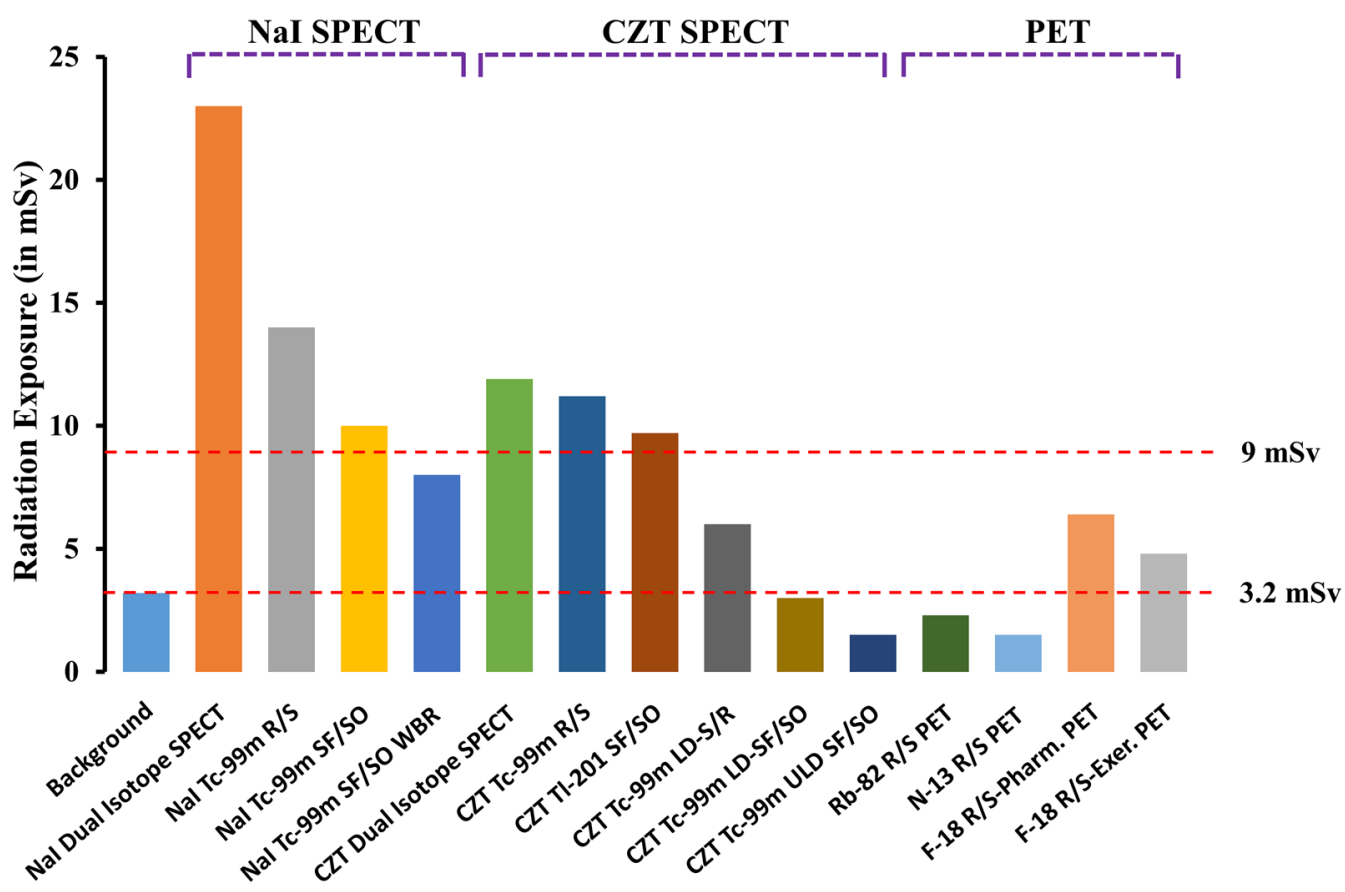

Figure 1. Approximate radiation effective doses from various SPECT and PET myocardial perfusion imaging protocols as reported in the literature. SPECT, single-photon emission computed tomography; $P E T$, positron emission tomography; $N a I$, sodium iodide; $R / S$, rest-stress; $S F$, stressfirst; $S O$, stress-only; $W B R$, wide beam reconstruction; $L D$, low dose; $U L D$, ultra-low dose; $S$ Pharm, pharmacologic stress; $S$-Exer, exercise stress. 
patients who had undergone ICA, Cramer et al. performed Tc-99m and Tl-201 imaging, in random order, and noted no clinically relevant differences in diagnostic accuracy between the two tracers. In another small study of 26 patients undergoing both Tc-99m and Tl-201 SPECT, there was a similar number of fixed defects identified by both tracers. ${ }^{15}$ Though Tl-201 identified a greater number of reversible defects, they were predominantly from regions with mild coronary artery disease. A more recent, larger study, of 163 patients with mild to moderate CAD (50\%-89\%) on ICA, compared the diagnostic performance of Tl-201 ( $n=69)$, Tc-99m sestamibi $(n=50)$ and Tc-99m tetrofosmin $(n=44){ }^{12}$ The authors reported no differences in the summed uptake scores between the three tracers and the percent of abnormal territories identified by them-Tl-201: $62 \%$ vs. Tc-99m sestamibi: $57 \%$ vs. Tc-99m tetrofosmin: $58 \%, P=0.8$, suggesting a comparable diagnostic value of all three tracers. While these studies were performed on conventional Anger camera, subgroup analysis of a more recent CZT-based study $(n=230)$ also showed no statistical difference in the diagnostic accuracy of Tc$99 \mathrm{~m}(68 \%)$ or $\mathrm{Tl}-201(71 \%)$ SPECT for anatomical CAD ( $\geq 70 \%$ stenosis). ${ }^{16}$

Maintaining laboratory efficiency is challenging, especially for nuclear medicine laboratories that also perform MPI. A higher throughput offered by a dual isotope protocol is certainly attractive in this regard. Though this may be true for conventional Anger systems, it does not fully harness the abilities of a CZT camera, wherein imaging can be completed within a few minutes with conventional rest and stress doses of Tc$99 \mathrm{~m}$. Laboratories where there are fewer constraints on throughput can perform low dose rest and stress imaging at significantly lower radiation exposure. Additionally, the rapidity with which imaging can be accomplished with CZT systems allows for low dose stress-first protocols to be implemented. This allows for rapid, accurate assessment of myocardial perfusion at a fraction of the conventional dose, with the ability to perform a 'higher dose' resting scan if stress-first is abnormal. Moreover, the need for performing a resting scan can be obviated by careful selection of patients (low risk) and subjecting them to a stress-only protocol. An algorithm of selection of such patients for CZT SPECT has been suggested by Duvall et. al. ${ }^{16}$

In this editorial, we provide a distribution of the approximate radiation exposure from a variety of MPI protocols reported in the literature (Figure 1). We strongly believe that though newer camera technology has greatly enhanced the field of nuclear cardiology, technological advancements alone are not sufficient to provide patient-centered imaging-appropriate and effective, with low radiation exposure. A laboratory should be armed with a gamut of imaging protocols (rest/stress, stress/rest, stress-first and stress-only, etc.) that should cater to the needs and demographics of patients being imaged. Careful preselection of patients is critical, and as shown in the figure, can result in significant radiation dose reduction, even with conventional Anger camera, with a greater impact with high-resolution CZT systems. Thus, given the lack of clinically significant difference in diagnostic accuracies of a dual isotope vs. single isotope protocol, the considerable improvement in image quality and the improved laboratory throughput conferred by solid-state detector systems, it would be regressive to implement a dual isotope protocol on a CZT camera.

\section{Disclosures}

Rami Doukky receives research funding grants from Astellas Pharma (Northbrook, IL) and serves on an advisory board for Astellas Pharma. Saurabh Malhotra has nothing to disclose.

\section{References}

1. Einstein AJ, Blankstein R, Andrews H, Fish M, Padgett R, Hayes $\mathrm{SW}$, et al. Comparison of image quality, myocardial perfusion, and left ventricular function between standard imaging and single-injection ultra-low-dose imaging using a high-efficiency SPECT camera: The MILLISIEVERT study. J Nucl Med. 2014;55:14307.

2. Einstein AJ, Pascual TN, Mercuri M, Karthikeyan G, Vitola JV, Mahmarian JJ, et al. Current worldwide nuclear cardiology practices and radiation exposure: Results from the 65 country IAEA Nuclear Cardiology Protocols Cross-Sectional Study (INCAPS). Eur Heart J. 2015;36:1689-96.

3. Imbert L, Roch V, Merlin C, Djaballah W, Cachin F, Perrin M et al. Low-dose dual-isotope procedure planed for myocardial perfusion CZT-SPECT and assessed through a head-to-head comparison with a conventional single-isotope protocol. J Nucl Cardiol. 2017.

4. Perrin M, Djaballah W, Moulin F, Claudin M, Veran N, Imbert L, et al. Stress-first protocol for myocardial perfusion SPECT imaging with semiconductor cameras: High diagnostic performances with significant reduction in patient radiation doses. Eur $\mathbf{J}$ Nucl Med Mol Imaging. 2015;42:1004-11.

5. van Dijk JD, Borren NM, Mouden M, van Dalen JA, Ottervanger JP, Jager PL. Effect of a patient-specific minimum activity in stress myocardial perfusion imaging using CZT-SPECT: Prognostic value, radiation dose, and scan outcome. J Nucl Cardiol. 2018;25:26-35.

6. Duvall WL, Croft LB, Godiwala T, Ginsberg E, George T, Henzlova MJ. Reduced isotope dose with rapid SPECT MPI imaging: Initial experience with a CZT SPECT camera. J Nucl Cardiol. 2010;17:1009-14.

7. Barone-Rochette G, Zoreka F, Djaileb L, Piliero N, Calizzano A, Quesada JL et al. Diagnostic value of stress thallium-201/rest technetium-99m-sestamibi sequential dual isotope high-speed 
myocardial perfusion imaging for the detection of haemodynamically relevant coronary artery stenosis. J Nucl Cardiol. 2018.

8. Henzlova MJ, Duvall WL, Einstein AJ, Travin MI, Verberne HJ. ASNC imaging guidelines for SPECT nuclear cardiology procedures: Stress, protocols, and tracers. J Nucl Cardiol. 2016;23:60639.

9. Glover DK, Ruiz M, Edwards NC, Cunningham M, Simanis JP, Smith WH, et al. Comparison between $201 \mathrm{Tl}$ and $99 \mathrm{mTc}$ sestamibi uptake during adenosine-induced vasodilation as a function of coronary stenosis severity. Circulation. 1995;91:813-20.

10. Leon AR, Eisner RL, Martin SE, Schmarkey LS, Aaron AM, Boyers AS, et al. Comparison of single-photon emission computed tomographic (SPECT) myocardial perfusion imaging with thallium-201 and technetium-99m sestamibi in dogs. J Am Coll Cardiol. 1992;20:1612-25.

11. Narahara KA, Villanueva-Meyer J, Thompson CJ, Brizendine M, Mena I. Comparison of thallium-201 and technetium-99m hexakis 2methoxyisobutyl isonitrile single-photon emission computed tomography for estimating the extent of myocardial ischemia and infarction in coronary artery disease. Am J Cardiol. 1990;66:1438-44.

12. Reyes E, Loong CY, Harbinson M, Rahman S, Prvulovich E, Ell PJ, et al. A comparison of Tl-201, Tc-99m sestamibi, and Tc-99m tetrofosmin myocardial perfusion scintigraphy in patients with mild to moderate coronary stenosis. J Nucl Cardiol. 2006;13:488-94.

13. Taillefer R, Dupras G, Sporn V, Rigo P, Leveille J, Boucher P, et al. Myocardial perfusion imaging with a new radiotracer, technetium-99m-hexamibi (methoxy isobutyl isonitrile): Comparison with thallium-201 imaging. Clin Nucl Med. 1989;14:89-96.

14. Cramer MJ, Verzijlbergen JF, Van der Wall EE, Niemeyer MG, Zwinderman AH, Ascoop CA, et al. Head-to-head comparison between technetium-99m-sestamibi and thallium-201 tomographic imaging for the detection of coronary artery disease using combined dipyridamole-exercise stress. Coron Artery Dis. 1994;5:787-91.

15. Shanoudy H, Raggi P, Beller GA, Soliman A, Ammermann EG, Kastner RJ, et al. Comparison of technetium-99m tetrofosmin and thallium-201 single-photon emission computed tomographic imaging for detection of myocardial perfusion defects in patients with coronary artery disease. J Am Coll Cardiol. 1998;31:331-7.

16. Duvall WL, Sweeny JM, Croft LB, Barghash MH, Kulkarni NK, Guma KA, et al. Comparison of high efficiency CZT SPECT MPI to coronary angiography. J Nucl Cardiol. 2011;18:595-604. 